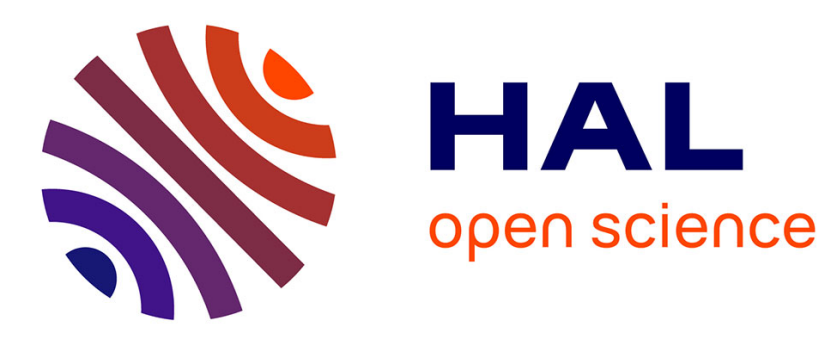

\title{
Global Production Increased by Spatial Heterogeneity in a Population Dynamics Model
}

Jean-Christophe Poggiale, Pierre Auger, David Nerini, Claude Manté, Franck Gilbert

\section{- To cite this version:}

Jean-Christophe Poggiale, Pierre Auger, David Nerini, Claude Manté, Franck Gilbert. Global Production Increased by Spatial Heterogeneity in a Population Dynamics Model. Acta Biotheoretica, 2005, vol. 53, pp. 359-370. 10.1007/s10441-005-4890-3 . hal-00755916

\section{HAL Id: hal-00755916 https://hal.science/hal-00755916}

Submitted on 22 Nov 2012

HAL is a multi-disciplinary open access archive for the deposit and dissemination of scientific research documents, whether they are published or not. The documents may come from teaching and research institutions in France or abroad, or from public or private research centers.
L'archive ouverte pluridisciplinaire HAL, est destinée au dépôt et à la diffusion de documents scientifiques de niveau recherche, publiés ou non, émanant des établissements d'enseignement et de recherche français ou étrangers, des laboratoires publics ou privés. 


\title{
GLOBAL PRODUCTION INCREASED BY SPATIAL HETEROGENEITY IN A POPULATION DYNAMICS MODEL
}

\author{
J.-C. Poggiale ${ }^{1}$, P. Auger ${ }^{2}$, D. Nérini ${ }^{1}$, C. Manté $^{1}$ \\ and F. Gilbert ${ }^{1}$
}

${ }^{1}$ Centre d'Océanologie de Marseille-UMR CNRS 6117 - LMGEM, Case 901 -Campus de Luminy, 13288 Marseille Cedex, France

${ }^{2}$ Institut de Recherche pour le Développement (IRD) - UR GEODES 32, avenue Henri Varagnat, 93143 Bondy Cedex, France

\begin{abstract}
Spatial and temporal heterogeneity are often described as important factors having a strong impact on biodiversity. The effect of heterogeneity is in most cases analyzed by the response of biotic interactions such as competition of predation. It may also modify intrinsic population properties such as growth rate. Most of the studies are theoretic since it is often difficult to manipulate spatial heterogeneity in practice. Despite the large number of studies dealing with this topics, it is still difficult to understand how the heterogeneity affects populations dynamics. On the basis of a very simple model, this paper aims to explicitly provide a simple mechanism which can explain why spatial heterogeneity may be a favorable factor for production. We consider a two patch model and a logistic growth is assumed on each patch. A general condition on the migration rates and the local subpopulation growth rates is provided under which the total carrying capacity is higher than the sum of the local carrying capacities, which is not intuitive. As we illustrate, this result is robust under stochastic perturbations.
\end{abstract}

\section{INTRODUCTION}

Heterogeneity has been recognized for a long time, in theoretical works, as a major factor affecting population dynamics (Levin, 1976; Hanski, 1981). Nowadays, there are more and more empirical evidences showing that spatial or temporal heterogeneities affect the functioning of ecosystems (Cardinale et al., 2002). However, it still remains difficult to understand how spatial variabilities of physical and chemical environmental conditions may modify the functional roles of living organisms. Various effects are usually envisioned and among them are the increase of growth of a population (Auger and Poggiale, 1996b; Poggiale, 1998), the possible explanations for the paradox of plankton (Huissman and Weissing, 1999) and for the paradox of enrichment (Luckinbill, 1974; Kooi et al., 1998) and more generally the fact that heterogeneity may lead to an increased biodiversity (Pacala and Roughgarden, 1982). More precisely, heterogeneity induces variabilities in the manner that individuals can feed or absorb there resources and these changes at the individuals scale may affect the population growth in a positive or a negative way. For instance, the interaction between a prey population and its predator can exhibit various response to the variability of environment induced by 
spatial or temporal heterogeneity (Bernstein et al., 1999; Scheffer and de Boer, 1995). The functional response of a such interaction is known to be sensible to heterogeneity as it has been shown in experimental and theoretical works (Arditi et al., 1991; Arditi and Saïah, 1992).

The impact of spatial or temporal heterogeneity on biodiversity is of great interest nowadays since many human activities tend to decrease the heterogeneities, which could result in a loss of biodiversity. Consequently, there is a urgent need of progress in the understanding of the response of living populations to perturbations of heterogeneities. Since it is not easy to manipulate spatial heterogeneity in experiments, lots of works are based on modelling. Indeed, this approach allows to test some assumptions rather easily. Moreover, a very simple model allowing detailed calculation permit also to understand precisely the mechanism underlying the possibility of an increased production. In this context, the present paper aims to provide a simple mathematical example from which the exact mechanism can be extracted, as described hereafter. Let us consider a population living in an habitat constituted by two patches, in which production rates vary according to the spatial location leading to a spatial heterogeneity. The individuals displace in this habitat, changing from one location to the other one. There are many natural examples which can be approximated by a such simplification. Among them, we suggest the zooplankton diel migration along the column water (Andersen, 1991). In this example, the individuals can eat the phytoplankton produced during the day at the surface layer and hide from their own predators in the bottom layer. We can conclude that the surface layer corresponds to a higher productive layer since food is available there. The second main assumption of the mechanism is that the individuals move preferentially from the good patch to the bad one. This behavior may for instance be explained by a high predation pressure on the good patch, leading the individuals to leave. The individuals come back on the good patch in order to feed. It follows from these assumptions, according to our model, that individuals are produced faster on the patch where they are less abundant. Since they are less abundant, the carrying capacity may be reached after a longer time, which leads to a higher production of individuals.

The paper is organized as follows. In the next section, we briefly explain the aggregation techniques which shall facilitate the study of the model and references are given for more details. Then we describe in details the model which will be used hereafter. The following section is devoted to the derivation of conditions for which the total number of individuals reached at equilibrium is higher when heterogeneity taken into account than in the homogeneous case. Next, we perform a numerical analysis to check if our result is robust under two types of stochastic perturbations which represent (i) environmental fluctuations and (ii) intrinsic individual variability. We end the paper by a short conclusion.

\section{AGGREGATION METHODS: TO DEAL WITH ECOLOGICAL COMPLEXITY}

Aggregation methods have been introduced in ecology by Iwasa et al. (1987, 1989). It concerns the possibility to write simplified models when the level of organization of interest is changed. For instance, at the ecosystem level, it is maybe not necessary to deal with all the details describing the individuals levels. In this case, the detailed description is made by microvariables and the aim is to describe the macrolevel with 
macrovariables. Generally, since we omit details, the number of macrovariables is much lower than the number of microvariables. The aggregation methods permit to derive objectives relationships between these variables (Auger and Poggiale, 1996a; Sanz and Bravo de la Parra, 1998)

In Auger and Poggiale 1998 and Poggiale and Auger (1996), the authors provide and illustrate a method based on Geometrical Singular Perturbation Theory and a theorem of reduction given by Fenichel (1971, refer also to Wiggins, 1994), refer to Appendix A for more details. Our version is based on the paper from Sakamoto (1990).

\section{DESCRIPTION OF THE MODEL}

We consider a two patches model on which the population grows logistically. Let $N_{i}$ be the amount of individuals on patch $i, r_{i}$ is the intrinsic growth rate of the population on patch $i$ and $K_{i}$ is the corresponding carrying capacity. Individuals can move randomly from patch to patch and let us denote $m_{i j}$ as the displacement rate from patch $j$ to patch $i$. Furthermore, we assume that the displacement of individuals are much faster than the demographic process. This assumption is described in the model by the mean of a small dimensionless parameter $\varepsilon$, so called time scale parameter. The model then reads as follows:

$$
\begin{aligned}
& \frac{d N_{1}}{d \tau}=m_{12} N_{2}-m_{21} N_{1}+\varepsilon r_{1} N_{1}\left(1-\frac{N_{1}}{K_{1}}\right) \\
& \frac{d N_{2}}{d \tau}=m_{21} N_{1}-m_{12} N_{2}+\varepsilon r_{2} N_{2}\left(1-\frac{N_{2}}{K_{2}}\right)
\end{aligned}
$$

We denote $N$ the total population density: $N=N_{1}+N_{2}$. If we first assume that the displacements do not occur in this system (case $m_{12}=m_{21}=0$ ), then the subpopulations exhibit logistic growth. After a transient time, the abundance of the subpopulation on patch $i$ reaches an equilibrium value $K_{i}$. As a consequence, the total number $N$ of individuals shall reach the value $K_{1}+K_{2}$. For the sake of simplicity, we assume in this paper that $K_{1}=K_{2}$. We show in the next section that, when the displacements occur, the total number of individuals reaches a larger value than $2 K_{1}$, provided that the subpopulations intrinsic growth rates are not the same on each patch $\left(r_{1} \neq r_{2}\right)$, that is the global system is spatially heterogeneous.

\section{CONDITIONS FOR AN INCREASED PRODUCTION}

\subsection{Reduction of the Model to a One Dimensional Logistic Equation}

We now take advantage of the different time scales to simplify the previous two dimensional system into a one dimensional model which exhibits the same dynamics for the total population abundance $N$. Since the displacement are fast, the number of individuals on each patch rapidly reaches a fixed proportion of the total number of individuals in the population. We denote by $u_{i}$ the corresponding proportion on patch $i$, that is $u_{i}=N_{i} / N$. Thus the amount of individuals on each patch reads:

$$
\begin{aligned}
& N_{1}^{*}=\frac{m_{12}}{m_{12}+m_{21}} N=u_{1} N \\
& N_{2}^{*}=\frac{m_{21}}{m_{12}+m_{21}} N=u_{2} N
\end{aligned}
$$


This equilibrium is obtained by vanishing the differential equations when $\varepsilon=0$. It permits to build a one dimensional model for the total population

$$
\frac{d N}{d \tau}=\varepsilon\left(r_{1} u_{1} N\left(1-\frac{u_{1} N}{K_{1}}\right)+r_{2} u_{2} N\left(1-\frac{u_{2} N}{K_{2}}\right)\right)
$$

This model can be written as a logistic equation:

$$
\frac{d N}{d t}=r N\left(1-\frac{N}{K}\right)
$$

where $t=\varepsilon \tau$ is a change of time unit and the parameters $r$ and $K$ are defined as follows:

$$
r=r_{1} u_{1}+r_{2} u_{2} \quad \text { and } \quad K=\frac{K_{1} K_{2}\left(r_{1} u_{1}+r_{2} u_{2}\right)}{K_{2} r_{1} u_{1}^{2}+K_{1} r_{2} u_{2}^{2}}
$$

For the sake of simplicity, we shall consider the particular case where $K_{1}=K_{2}$. The more general case would lead to similar results but with more calculations. This assumption permits to simplify the total carrying capacity of the domain, that is the maximum value reachable by the number of individuals.

$$
K=\frac{K_{1}\left(r_{1} u_{1}+r_{2} u_{2}\right)}{r_{1} u_{1}^{2}+r_{2} u_{2}^{2}}
$$

\subsection{Properties of Spatial Homogeneity: $r_{1}=r_{2}$}

Let us first consider the case of a homogeneous environment, $r_{1}=r_{2}$. It then follows that $r=r_{1}$ and $K=K_{1} /\left(u_{1}^{2}+u_{2}^{2}\right)$. Since $u_{1}^{2}+u_{2}^{2}$ is a number comprised between 0.5 and 1 when $u_{1}$ is a proportion, it follows that $K \leq 2 K_{1}$. There is a particular case when the displacement rates $m_{12}$ and $m_{21}$ are equal. Indeed, in this case, the subdivision of the population into two subpopulations is arbitrary and this leads to $K=2 K_{1}$, that is there is no effect. However, if the displacement rates are not the same, then the total carrying capacity is lower than the sum of the local carrying capacity. In the following subsection, we show that this result can be reversed when the growth rates on each patch are not the same, that is in the heterogeneous case. Moreover, the range of the displacements parameters where the total carrying capacity is higher than the sum of the local carrying capacities is enlarged when the heterogeneity is stronger.

\subsection{Increased Production by Spatial Heterogeneity: $r_{1} \neq r_{2}$}

We prove here that when the environment is heterogeneous, $K$ can be higher than $2 K_{1}$. As it can be shown by a straightforward calculation (see appendix B), $K$ is larger than $2 K_{1}$ if and only if the following inequality is satisfied:

$$
2\left(r_{1}+r_{2}\right) u_{1}^{2}-\left(3 r_{2}+r_{1}\right) u_{1}+r_{2}<0
$$

The left-hand side of this inequality is a quadratic term with respect to the proportion of individuals in patch $1, u_{1}$. This quadratic term must be negative which means that $u_{1}$ must be between the roots of the associated quadratic equation. The corresponding roots are:

$$
u_{1}^{+}=\frac{3 r_{2}+r_{1}+\sqrt{\left|r_{1}-r_{2}\right|}}{4\left(r_{1}+r_{2}\right)} \quad \text { and } \quad u_{1}^{-}=\frac{3 r_{2}+r_{1}-\sqrt{\left|r_{1}-r_{2}\right|}}{4\left(r_{1}+r_{2}\right)}
$$


Notice that the absolute values of the displacements rates are not really important for our study, since only $m_{12} / m_{21}$ appears in the calculations, by the mean of $u_{1}=\frac{1}{1+\frac{m_{12}}{m_{12}}}$. Thus only the ratio $m_{21} / m_{12}$ is of interest in our case. The range of the parameter $u_{1}$ for which the production is increased by heterogeneity is:

$$
u_{1}^{+}-u_{1}^{-}=\frac{\left|r_{1}-r_{2}\right|}{2\left(r_{1}+r_{2}\right)}
$$

Thus, if $r_{1} \neq r_{2}$ then there exists $u_{1}$ such that $K>2 K_{1}$. Moreover, the range of the parameter $u_{1}$ for which this inequality holds increases with $\left|r_{1}-r_{2}\right|$. As a consequence, even if, in the heterogeneous case, we can have $K<2 K_{1}$, this inequality becomes rare in the case of strong heterogeneity. Since we are considering the case $r_{1} \neq r_{2}$, let us assume that $r_{1}>r_{2}$ (the opposite case would be completely similar). Then, we get:

$$
u_{1}^{+}=\frac{1}{2} \quad \text { and } \quad u_{1}^{-}=\frac{r_{2}}{r_{1}+r_{2}}
$$

It follows that the total carrying capacity is larger than the sum of the local carrying capacities if and only if we have the following inequalities:

which is equivalent to:

$$
\frac{r_{2}}{r_{1}+r_{2}}<u_{1}=\frac{m_{12}}{m_{12}+m_{21}}<\frac{1}{2}
$$

$$
1<\frac{m_{21}}{m_{12}}<\frac{r_{1}}{r_{2}}
$$

This last inequalities show that (i) the total carrying capacity can be enlarged only if the local growth rates are different and (ii) the carrying capacity is enlarged when the ratio of the displacement rate from patch 1 to patch 2 over the displacement rate from patch 2 to patch 1 is lower than the ratio of the local growth rate $r_{1}$ over the local growth rate $r_{2}$.

Our result is illustrated on Figures 1 and 2. Figures 1 shows a comparison between the two dimensional model and the aggregated model. Figures 2 illustrates the enhancement of carrying capacity when the displacement of individuals occur. On this figure, we can see that the total carrying capacity is higher than the sum of the local ones. The parameter values for numerical simulations are given in Table 1.

\section{ROBUSTNESS OF THE RESULTS UNDER RANDOM PERTURBATIONS}

In this section, we randomly perturb the above dynamical system in order to check the robustness of the results. Two different kind of perturbations are considered. The first one is an additive white noise which simulates the environmental variability. The second one describes an individual variability through random growth rates.

\subsection{Environmental Variability}

The perturbed processes reads:

$$
\begin{aligned}
& d N_{1}=m_{12} N_{2} d \tau-m_{21} N_{1} d \tau+\varepsilon r_{1} N_{1}\left(1-\frac{N_{1}}{K_{1}}\right)+\delta_{1} d W_{1} \\
& d N_{2}=m_{21} N_{1} d \tau-m_{12} N_{2} d \tau+\varepsilon r_{2} N_{2}\left(1-\frac{N_{2}}{K_{2}}\right)+\delta_{2} d W_{2}
\end{aligned}
$$




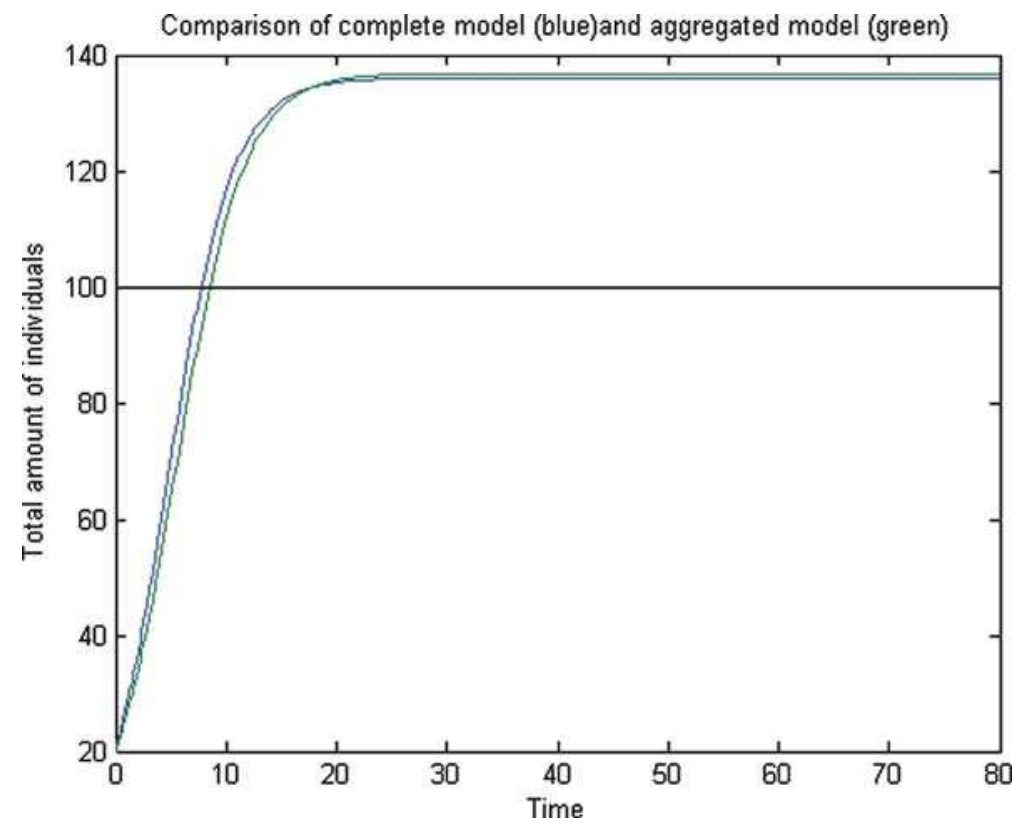

Figure 1. Comparison the aggregated model and the sum of the local densities obtained with the complete model. The blue curve illustrates the simulation with the aggregated model while the green one has been obtained by summing the variables solved from the complete two dimensional system. Both curves are very close when $\varepsilon$ is small enough. The parameter values are given in Table 1 .

where $W_{1}$ and $W_{2}$ are independent Wiener processes, and $\delta_{i}, i=1,2$ are small numerical parameters controlling the strength of the perturbation. We consider here the effect of small perturbations i.e. the perturbing effect induced by $\delta_{i} d W_{i}$ is small compared to the deterministic part of the system. The perturbation of the dynamical system by a Wiener process can be considered as an environmental variability that should affect the population dynamics. This perturbation does not modify the previous result (Figure 3). As it can be seen on this figure, the aggregated model still well approximates the total density of the complete perturbed model. This is the consequence of a known result shown by Berglund and Gentz (2003).

\subsection{Random Growth Rates}

Another way to introduce variability, is to consider random perturbations according to individuals variations themselves. In the present work, we considered that the individual variations can be described as perturbations of the growth rates. These rates $r_{i}, i=1,2$, are taken as real random variables with uniform distributions on supports $\left[a_{i} ; b_{i}\right] i=1,2$ such that the expectation of $r_{1}$ is larger than the expectation of $r_{2}$ :

$$
\begin{aligned}
& E\left(r_{1}\right)=10, \quad\left[a_{1} ; b_{1}\right]=[0 ; 20] \\
& E\left(r_{2}\right)=1, \quad\left[a_{2} ; b_{2}\right]=[0 ; 2]
\end{aligned}
$$




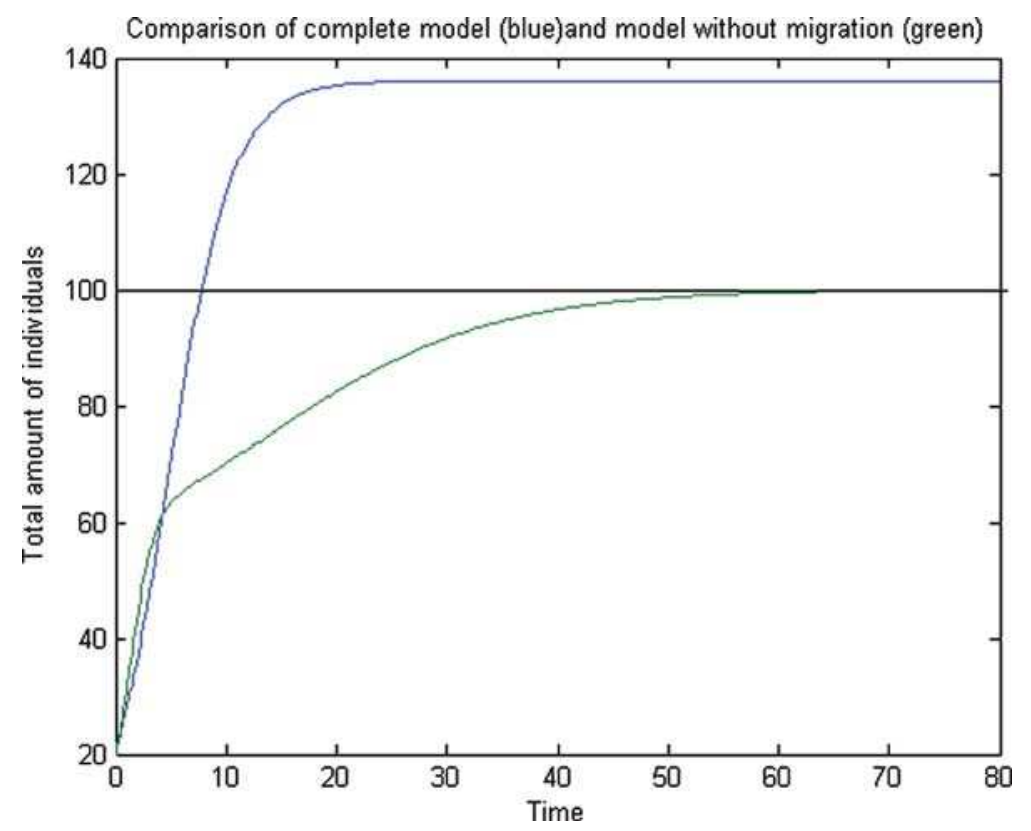

Figure 2. Comparison between the simulations obtained with and without migrations. The blue curve results from the complete model. The green curve is obtained by vanishing the migration rates in order to describe the dynamics resulting from the juxtaposition of two homogeneous environment and by summing the local densities. The total carrying capacity with migrations is higher that the sum of the local carrying capacities. The parameter values are given in Table 1 .

Table 1. List of parameters with their definitions, their notations and their numerical values used in the simulations

\begin{tabular}{llc}
\hline Definition of the parameter & $\begin{array}{l}\text { Name of the } \\
\text { parameter }\end{array}$ & $\begin{array}{l}\text { Value of the } \\
\text { parameter }\end{array}$ \\
\hline Growth rate of subpopulation 1 & $r_{1}$ & 10 \\
Growth rate of subpopulation 2 & $r_{2}$ & 1 \\
Carrying capacity on patch 1 & $K_{1}$ & 50 \\
Carrying capacity on patch 2 & $K_{2}$ & 50 \\
Migration rate from patch 1 to 2 & $m_{21}$ & 3 \\
Migration rate from patch 2 to 1 & $m_{12}$ & 1 \\
Time scale factor & $\varepsilon$ & 0.1 \\
Initial condition for density on patch 1 & $N_{1}(0)$ & 10 \\
Initial condition for density on patch 2 & $N_{2}(0)$ & 10 \\
\hline
\end{tabular}

The variances has been chosen in a such manner that the support of $r_{2}$ belongs to the support of $r_{1}$. This means that the probability that $r_{1} \leq r_{2}$ is not negligible. The other parameters are still the same as those given in table 1 . According to the previous section, when $r_{1} \leq r_{2}$ then the result fails, that is the total carrying capacity is smaller than the 


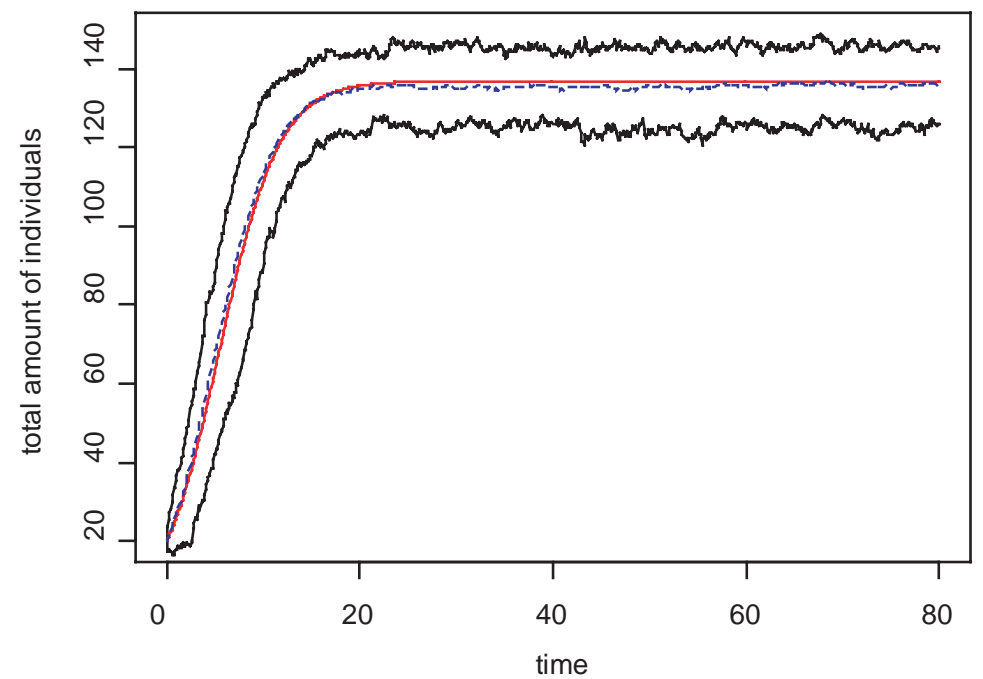

Figure 3. Comparison between the aggregated model and simulations of the random perturbed dynamical system (Wiener process, $\delta_{1}=\delta_{2}=3$ ). The red line draws the aggregated model. The black lines represent a variability interval calculated over 100 simulations. At time $t$, the lower bound is the 0.025 -quantile and the upper bound is the 0.975 -quantile. The blue dashed line is the mean trajectory of 100 simulations.

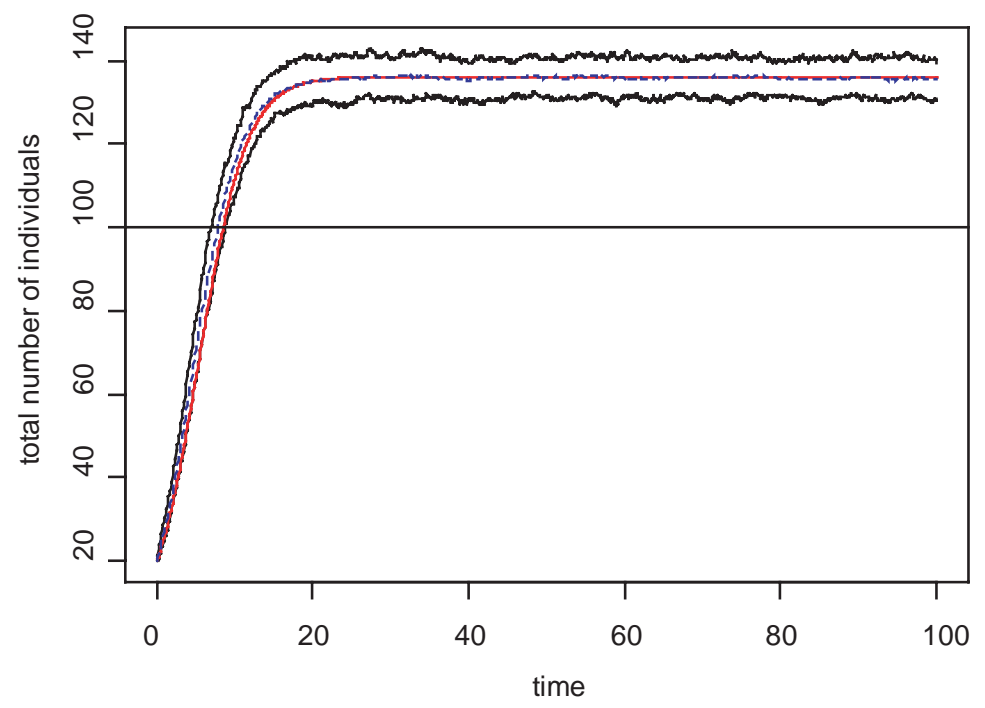

Figure 4. Comparison between the aggregated model and simulations of the random dynamical system when growth rates are random variables. The red line draws the aggregated model. The black lines represent a variability interval calculated over 100 simulations. At time $t$, the lower bound is the 0.025 quantile and the upper bound is the 0.975 -quantile. The blue dashed line is the mean trajectory of 100 simulations. 
sum of the local ones. Our numerical simulation aims to illustrate what happens when $r_{1}$ is sometimes lower than $r_{2}$ with random occurences. The result is illustrated on Figure 4 and shows that the solution of the aggregated model remains close to the total density obtained with the complete perturbed model.

\section{CONCLUSION}

In this paper, we have shown that the number of individuals in a heterogeneous environment may be larger than what it should be in an equivalent homogeneous environment. More precisely, the carrying capacity of an given environment may be enlarged by spatial heterogeneity. This has been shown on a particular simple model. The simplicity of our model permitted to deal with a complete description of the conditions under which our result is valid.

It is usually recognized that production in natural environment is larger than production obtained in experiments. However, it is really difficult to develop experiments to test our result. It is then necessary to develop theoretical tools to understand the effect of heterogeneity on biological processes. One of the interest of such tools can reside in their simplicity. Moreover, our explanations are based on the mathematical expressions obtained in the result derivation. We shown that in a homogeneous environment, the total carrying capacity is generally lower than the sum of the local carrying capacities while this result can be inverted when heterogeneity occurs. Furthermore, the range parameter for which the total carrying capacity is enlarged is larger when the heterogeneity is stronger.

Finally, we illustrated by numerical simulations that the previous result is robust under stochastic perturbations. Two different stochastic perturbations have been considered. The first one aimed to describe an environmental variability and is formulated by an additive white noise. The second one dealt with the individual variability of the growth rate and it has been described by a uniform random process added to the local growth rates. In both cases, we saw that the one dimensional not perturbed aggregated model is still a good approximation of the complete randomly perturbed model. It is known for the first case of perturbation (environmental variability). It is a purely numeric result in the second case.

\section{APPENDIX A: SLOW - FAST SYSTEMS}

Let us consider a slow - fast system:

$$
\begin{aligned}
& \frac{d x}{d t}=f(x, y, \varepsilon) \\
& \frac{d y}{d t}=\varepsilon g(x, y, \varepsilon)
\end{aligned}
$$

where $\varepsilon$ is a small parameter and $x$ and $y$ are vectors in $R^{k_{1}}$ and $R^{k_{2}}$ respectively. We assume that when $\varepsilon=0$, there exists an invariant manifold $M_{0}$ defined by the equilibrium of the fast part:

$$
M_{0}=\{(x, y) / f(x, y, 0)=0\}
$$

We assume as well that this manifold is normally hyperbolic, which means that the Jacobian matrix of the previous system at each point of $M_{0}$ has exactly $k_{2}$ eigenvalues 
on the imaginary axis. If furthermore the other eigenvalues have a negative real part, then $M_{0}$ is an attracting set. It follows from these assumptions that for $\varepsilon>0$ sufficiently small, there exists an invariant manifold $M_{\varepsilon}$ close and diffeomorph to $M_{0}$. The solutions of the previous model in the vicinity of $M_{\varepsilon}$ are close to the solutions of the restriction on $M_{\varepsilon}$. We thus consider the restriction on the manifold $M_{\varepsilon}$, which leads to a reduced dimension. This procedure provides the aggregated model.

Moreover, it can be shown (Berglund and Gentz, 2003) that, as long as the invariant manifold $M_{\varepsilon}$ is attracting, the trajectories starting in the vicinity of $M_{\varepsilon}$ stays around $M_{\varepsilon}$ for an exponentially long time. This means that the sum of the randomly perturbed equations of local densities may still be compared to the solution of the aggregated model.

\section{APPENDIX B: CONDITIONS FOR INCREASED CARRYING CAPACITY}

In this section, we derive a condition on $u_{1}$ which, when satisfied, is equivalent to $K>K_{1}+K_{2}$. Let us recall that $u_{2}=1-u_{1}$.

$$
\begin{aligned}
K>2 K_{1} & \Leftrightarrow \frac{r_{1} u_{1}+r_{2} u_{2}}{r_{1} u_{1}^{2}+r_{2} u_{2}^{2}}>2 \\
& \Leftrightarrow r_{1} u_{1}+r_{2} u_{2}>2\left(r_{1} u_{1}^{2}+r_{2} u_{2}^{2}\right) \\
& \Leftrightarrow r_{1} u_{1}+r_{2}-r_{2} u_{1}>2\left(r_{1} u_{1}^{2}+r_{2}-2 r_{2} u_{1}+r_{2} u_{1}^{2}\right) \\
& \Leftrightarrow 2\left(\left(r_{1}+r_{2}\right) u_{1}^{2}-2 r_{2} u_{1}+r_{2}\right)-\left(r_{1}-r_{2}\right) u_{1}-r_{2}<0 \\
& \Leftrightarrow 2\left(r_{1}+r_{2}\right) u_{1}^{2}-\left(3 r_{2}+r_{1}\right) u_{1}+r_{2}<0
\end{aligned}
$$

\section{APPENDIX C: RANDOM PERTURBATIONS}

Let:

$$
d N_{t}^{i}=f_{i}\left(N_{t}^{1}, N_{t}^{2}\right) d t+\delta^{i} d W_{t}^{i}, \quad i=1,2
$$

be a stochastic dynamical system, where $f_{i}, i=1,2$ are continuous functions of $\left(N_{t}^{1}, N_{t}^{2}\right), W^{i}, i=1,2$ are independent Wiener processes and $\delta^{i}, i=1,2$, are real numbers. Integrating the above equation on the time interval $[0 ; T]$, the solution takes the following form, which does not contain distributions:

$$
N_{t}^{i}=N_{0}^{i}+\int_{0}^{T} f_{i}\left(N_{s}^{1}, N_{s}^{2}\right) d s+\delta^{i}\left(W_{t}^{i}-W_{0}^{i}\right), \quad i=1,2
$$

It's proved that for arbitrary moments of time $t_{n}>t_{n-1}>\cdots>t_{1} \geq 0$, the random variables:

$$
\left(W_{t_{n}}-W_{t_{n-1}}\right),\left(W_{t_{n-1}}-W_{t_{n-2}}\right), \ldots,\left(W_{t_{2}}-W_{t_{1}}\right)
$$

have a joint Gaussian distribution and that these increments are independent. Moreover, we note that an increment $\left(W_{t}-W_{s}\right)$ from time $s$ to time $t, s<t$ has a Gaussian distribution with:

$$
E\left(W_{t}-W_{s}\right)=0, V\left(W_{t}-W_{s}\right)=E\left(W_{t}-W_{s}\right)^{2}=t-s
$$


where $E(\cdot)$ denotes the expectation. If $\delta^{i} \rightarrow 0$, the processes $N_{t}^{i}$ converge in probability to the solutions of the deterministic dynamical system, uniformly on every finite interval $[0, T]$, for Lipschitz conditions on $f_{i}$.

The integral of the solution can be solved by a suitable numerical integration scheme (Runge-Kutta scheme). At each step of the calculus, the numerical solution of the equation is perturbed by adding a real number drawn from a Gaussian distribution of variance $\delta^{2} \Delta t$, where $\delta^{2} \Delta t$ is the numerical time increment multiplied by the squared $\delta$ parameter.

\section{REFERENCES}

Andersen, V. and P. Nival (1991). A model of the diel vertical migration of zooplankton based on euphausiids. J. Marine Research 49: 153-175.

Arditi, R. and H. Saïah (1992). Empirical evidence of the role of heterogeneity in ratio-dependent consumption. Ecology 73(5): 1544-1551.

Arditi, R., N. Perrin and H. Saïah (1991). Functional responses and heterogeneities: An experimental test with cladocerans. Oikos 60: 69-75.

Auger, P. and J.C. Poggiale (1998). Aggregation and emergence in systems of ordinary differential equations. Mathematical Computer Modelling 27(4): 1-22.

Auger, P. and J.C. Poggiale (1996a). Reduction of complexity and emergence in hierarchically organized systems: Population dynamics. Systems Analysis Modelling Simulation 26: 217237.

Auger, P. and J.C. Poggiale (1996b). Emergence of population growth models: Fast migration and slow growth. Journal of Theoretical Biology 182: 99-108.

Berglund, N. and B. Gentz (2003). Geometric singular perturbation theory for stochastic differential equations. Journal of Differential Equations 191: 1-54.

Bernstein, C., P.M. Auger and J.C. Poggiale (1999). Predator Migration decisions, the Ideal Free Distribution and predator-prey dynamics. The American Naturalist 153(3): 267-281.

Bravo de la Parra, R., E. Sanchez and P. Auger (1997). Time scales in density dependent discrete models. J. of Biological Systems 5(1): 111-129.

Cardinale, B.J., M.A. Palmer, C.M. Swan, S. Brooks and N. Leroy Poff (2002). The influence of substrate heterogeneity on biofilm metabolism in a stream ecosystem. Ecology 83(2): 412-422.

Fenichel, N. (1971). Persistence and smoothness of invariant manifolds for flows. Indiana University Mathematics Journal 21(3): 193-226.

Hanski, I. (1981). Coexistence of competitors in patchy environments with and without predation. Oikos 37: 306-312.

Huissman, J. and F.J. Weissing (1999). Biodiversity of plankton by species oscillations and chaos. Nature 402: 407-410.

Iwasa, Y., V. Andreasen and S. Levin (1987). Aggregation in model ecosystems. I. Perfect Aggregation. Ecological Modeling 37: 287-302.

Iwasa, Y., S. Levin and V. Andreasen (1989). Aggregation in model ecosystems. II. Approximate Aggregation. Journal of Mathematics Applied in Medicine and Biology 6: 1-23.

Kooi, B.W., J.C. Poggiale and P.M. Auger (1998). Aggregation methods in food chains. Mathematical Computer Modelling 27(4): 109-120.

Levin, S.A. (1976). Population dynamics models in heterogeneous environments. Annual Review of Ecology and Systematics 7: 287-310.

Luckinbill, L.S. (1974). The effects of space and enrichment on a predator-prey system. Ecology 55: $1142-1147$. 
Pacala, S.W. and J. Roughgarden (1982). Spatial heterogeneity and interspecific competition. Theoretical Population Biology 121: 92-113.

Poggiale, J.C. (1998). From Behavioral to Population level: Growth and competition. Mathematical Computer Modelling 27(4): 41-50.

Poggiale, J.C. and P. Auger (1996). Fast Oscillating Migrations in a predator-prey model. Mathematical Models and Methods in Applied Sciences 6(2): 217-226.

Sakamoto, K. (1990). Invariant manifolds in singular perturbations problems for ordinary differential equations. Proc. Roy. Soc. Ed. 116A: 45-78.

Sanz, L. and R. Bravo de la Parra (1998). Variables aggregation in time varying discrete systems. Actabiotheoretica 46: 273-297.

Scheffer, M. and R.J. De Boer (1995). Implications of spatial heterogeneity for the paradox of enrichment. Ecology 76(7): 2270-2277.

Wiggins, S. (1994). Normally Hyperbolic Invariant Manifolds in Dynamical Systems, AMS 105, Springer-Verlag. 